\section{LACC1 Gene Defects in Familial Form of Juvenile Arthritis}

\section{To the Editor:}

Juvenile idiopathic arthritis [JIA; Mendelian Inheritance in Man (MIM) 604302] is the most common chronic childhood arthritis, characterized by chronic articular findings of unknown origin, with heterogeneity in disease course and systemic involvement ${ }^{1,2}$. Epidemiologic studies based on different diagnostic criteria showed varying prevalence from 0.07 to 4.01 per 1000 children across populations, an increased risk for European descendants, and different subtype distributions among ethnic groups ${ }^{3,4}$. JIA is generally known as a complex genetic trait with non-Mendelian inheritance pattern possibly resulting from interactions of multiple genetic loci and environmental factors ${ }^{5}$. However, several studies have reported causative variants in the Laccase (multicopper oxidoreductase) domain-containing 1 (LACC1; MIM 613409) gene in rare familial forms $\mathrm{s}^{6,7,8}$.

We present 17 patients (10 males and 7 females) from 7 families (5 with known parental consanguinity), each with 2-4 affected members diagnosed with JIA. This study was approved by the Institutional Review Board of Istanbul Technical University (MBG.22/2014) and carried out in compliance with the Declaration Helsinki.

Clinical and laboratory findings are summarized in Table 1. Mean age 1of patients was $15.3 \pm 9.1$ (range 2.8-44) years and mean disease duration 11.9 years. In 12 patients, disease onset was before age 3 . Subtype of arthritis was polyarticular in 13 patients, enthesitis-related in 3 patients, and oligoarticular in 1 patient. The disease was episodic in 6 of the patients, whereas it was chronic in the others. Ten patients still had active disease and 11 were still under biologic therapy.

During the course of the disease, large joints were involved in all patients except B III, and in 9 of them, small joints were also involved. Join involvement was symmetrical in 13 patients. Flexion or extension contractures developed at joints in 8 patients. No patient had gastrointestinal symptoms such as abdominal pain, vomiting, diarrhea, or oral/anal aphthous lesions that are suggestive of inflammatory bowel disease.

We first performed multipoint linkage analysis using high-density single-nucleotide polymorphism (SNP) genotyping microarrays (Illumina OmniExpress-24 BeadChip) in 2 families (A and B). A maximal cumulative LOD score of 4.81 was obtained at 13q13.3-13q14.13. Overlapping homozygosity at the linked locus was $6.8 \mathrm{Mb}$ and harbored 95 genes, 38 of which were protein-coding, including the $L A C C 1$. Because of the possibility of association between rare variants in $L A C C 1$ and familial form of JIA, we prioritized $L A C C 1$ for sequencing. In both families we found novel/rare, pathogenic/likely pathogenic variants segregating with the disease by Sanger

Table 1A. Demographic and clinical features of patients with novel/rare homozygous $L A C C 1$ variant.

\begin{tabular}{|c|c|c|c|c|c|c|c|c|c|}
\hline \multirow{2}{*}{$\begin{array}{l}\text { Variables } \\
\text { Patient }\end{array}$} & \multicolumn{4}{|c|}{ Family A } & \multicolumn{3}{|c|}{ Family B } & \multicolumn{2}{|c|}{ Family C } \\
\hline & AI & AII & AIII & AIV & BI & BII & BIII & CI & CII \\
\hline Age, yrs & 44 & 19 & 18 & 16 & 19 & 16 & 5 & 23 & 10 \\
\hline Age at onset, yrs & $<1$ & $<1$ & 2 & $<1$ & 2.5 & $<1$ & $<1$ & $<1$ & 2 \\
\hline Disease duration, yrs & 43.5 & 18.5 & 16 & 15.6 & 16.5 & 15.3 & 4.3 & 22.3 & 8 \\
\hline Type of onset & Poly- & Poly- & Poly- & Poly- & Poly- & Poly- & Poly- & Systemic & Systemic \\
\hline Remission* & + & + & - & + & + & - & - & - & - \\
\hline Fever/rash & $-1-$ & $-1-$ & $+1-$ & - & $-1-$ & $+/-$ & $-1-$ & $+/+$ & $+/+$ \\
\hline Organ involvement & - & - & - & - & Splenomegaly & Splenomegaly & - & Pericarditis & - \\
\hline Growth retardation & + & - & - & - & - & - & - & $+($ severe $)$ & $+($ severe $)$ \\
\hline Consanguinity & Yes & Yes & Yes & Yes & Yes & Yes & Yes & Yes & Yes \\
\hline$L A C C 1$ variants & Hom p. 0 & Hom p.0 & Hom p.0 & Hom p.0 & $\begin{array}{c}\text { Hom p. } \\
\text { (Arg414Ter) }\end{array}$ & $\begin{array}{c}\text { Hom p. } \\
\text { (Arg414Ter) }\end{array}$ & $\begin{array}{c}\text { Hom p. } \\
\text { (Arg414Ter) }\end{array}$ & $\begin{array}{l}\text { Hom p. } \\
\text { (Ile330del) }\end{array}$ & $\begin{array}{l}\text { Hom p. } \\
\text { (Ile330del) }\end{array}$ \\
\hline
\end{tabular}

Table $1 B$. Demographic and clinical features of patients without novel/rare homozygous $L A C C 1$ variant.

\begin{tabular}{|c|c|c|c|c|c|c|c|c|}
\hline \multirow{2}{*}{$\begin{array}{l}\text { Variables } \\
\text { Patient }\end{array}$} & \multicolumn{2}{|c|}{ Family D } & \multicolumn{2}{|c|}{ Family E } & \multicolumn{2}{|c|}{ Family F } & \multicolumn{2}{|c|}{ Family G } \\
\hline & DI & DII & EI & EII & FI & FII & GI & GII \\
\hline Age, yrs & 17.5 & 14 & 10.3 & 2.8 & 15 & 10 & 14.5 & 6.5 \\
\hline Age at onset, yrs & 15 & 1.5 & 2.5 & $<1$ & 10 & 7 & 8 & 5 \\
\hline Disease duration, yrs & 2.5 & 12.5 & 9.8 & 2.5 & 5 & 3 & 6.5 & 1.5 \\
\hline Type of onset & ERA & Oligo- & Poly- & Poly- & ERA & Oligo- & Poly - & Poly- \\
\hline Remission* & - & - & - & - & + & + & + & - \\
\hline Fever/rash & $-1-$ & $-1-$ & $-1-$ & $-1-$ & $-1-$ & $-1-$ & $-1-$ & $-1-$ \\
\hline Organ involvement & - & - & - & - & - & - & - & - \\
\hline Growth retardation & - & - & - & - & - & - & - & - \\
\hline Consanguinity & No & No & Yes & Yes & Yes & Yes & No & No \\
\hline$L A C C 1$ variants & No variant & No variant & No variant & No variant & $\begin{array}{c}\text { Hom p. } \\
\text { (Ile254Val) + } \\
\text { Het p.(Cys370Tyr) }\end{array}$ & $\begin{array}{c}\text { Hom p. } \\
\text { (Ile254Val) + } \\
\text { Het p.(Cys370Tyr) }\end{array}$ & $\begin{array}{c}\text { No } \\
\text { variant }\end{array}$ & No variant \\
\hline
\end{tabular}

${ }^{*}$ Remission criteria are defined according to the study of Wallace, et al (2004) ${ }^{9}$. Hom: homozygous; Het: heterozygous; ERA: enthesitis-related arthritis; oligo-: oligoarticular; poly-: polyarticular. 
sequencing of the coding regions and exon-intron junctions of LACC1. Novel variant c. $3 \mathrm{G}>\mathrm{A}(\mathrm{p} .0)$ in family $\mathrm{A}$ is deduced to disrupt the translational initiation codon (Figure 1), and Western analysis showed absence of protein production (data not shown). Rare variant c.1240C $>\mathrm{T}$ [p.(Arg414Ter); rs 184370809] in family B resulted in premature termination at codon 414, causing the deletion of the terminal 17 amino acids. Our findings verified that $L A C C 1$ gene defects underlie the familial form of juvenile arthritis and prompted us to sequence the coding regions of $L A C C 1$ in the remaining 5 families. Some other rare variants were detected in 2 families. Homozygous variant c.988_990del [p.(Ile330del); rs776489319] in family C is an in-frame deletion that resulted in the absence of the isoleucine residue at position 330 . Patients of family F carried a common homozygous variant c.760A $>\mathrm{G}$ [p.(Ile254Val); rs3764147] together with novel heterozygous c.1109G $>\mathrm{A}$ [p.(Cys370Tyr)]; however, an unaffected brother (F III) also had the same genotype, indicating that the 2 variants are not sufficient to cause juvenile arthritis on their own but could be susceptibility alleles. Patients in the remaining 3 families (D, E, G) did not carry any rare/novel variants in LACC1.

Further, the 2 patients in family E, which was the only consanguineous family without a possible causative $L A C C 1$ variant, were also subjected to SNP genotyping to investigate whether they shared genotypes in the LACC1 region. SNP genotypes in the $L A C C 1$ region were different, excluding shared compound heterozygosity and indicating that $L A C C 1$ was not the causative gene in this family.

Our findings confirm that $L A C C 1$ variants can be responsible for the recessive form of juvenile arthritis. Age of onset appears to be earlier in patients with $L A C C 1$ pathogenic/likely pathogenic variants. The finding that affected siblings in family $\mathrm{E}$ do not share genotypes indicates genetic heterogeneity in familial juvenile arthritis for the first time, to our knowledge, assuming Mendelian inheritance. Future genetic studies to assess the contribution to JIA of variants in $L A C C 1$ could clarify whether $L A C C 1$ variants underlie early onset JIA as in all reported cases. Genome-wide studies are essential to understand the contribution of genetic factors to the disease.

Our findings verify that $L A C C 1$ pathogenic variants can cause familial juvenile arthritis. Because juvenile arthritis has high clinical variability, as observed here even within the same family, we propose that screening patients with JIA, especially those with very early onset, for $L A C C 1$ variants can be beneficial to patients and families.

İLKER KARACAN, MSc, Molecular Biology, Biotechnology and Genetics Department, Dr. Orhan Öcalgiray Molecular Biology-Biotechnology and Genetics Research Centre, Graduate School of Science, Engineering and Technology, Istanbul Technical University; SERDAL UĞURLU, MD, Division of Rheumatology, Cerrahpaşa Medical Faculty, Istanbul University; SEZGIN ŞAHIN, MD, Department of Paediatric Rheumatology, Cerrahpaşa Medical School, Istanbul University; ELIF EVEREST, MSc, Molecular Biology, Biotechnology and Genetics Department, Dr. Orhan Öcalgiray Molecular Biology-Biotechnology and Genetics Research Centre, Graduate School of Science, Engineering and Technology, Istanbul Technical University; ÖZGÜR KASAPÇOPUR, MD Department of Paediatric Rheumatology, Cerrahpaşa Medical School, Istanbul University; ASLIHAN TOLUN, PhD, Department of Molecular Biology and Genetics, Boğaziçi University; HURI ÖZDOĞAN, MD, Division of Rheumatology, Cerrahpaşa Medical Faculty, Istanbul University; EDA TAHIR TURANLI, PhD, Molecular Biology, Biotechnology and Genetics Department, Dr. Orhan Öcalgiray Molecular Biology-Biotechnology and Genetics Research Centre, Graduate School of Science, Engineering and Technology, Istanbul Technical University, and Department of Molecular Biology and Genetics, Istanbul Technical University, Istanbul, Turkey. This study was supported by the Technological and Scientific Research Council of Turkey (Grant 114Z829). Address correspondence to Dr. E. Tahir Turanl, Dr. Orhan Öcalgiray Molecular Biology-Biotechnology and Genetics Research Centre, Istanbul Technical University, Ayazağa Campus, 34469 Maslak, Istanbul,Turkey.E-mail: turanlie@itu.edu.tr

\section{REFERENCES}

1. Petty RE, Southwood TR, Manners P, Baum J, Glass DN, Goldenberg J, et al; International League of Associations for Rheumatology. International League of Associations for
Family A

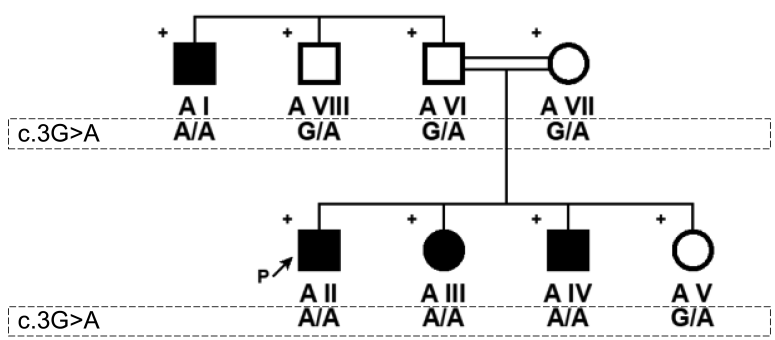

Family C

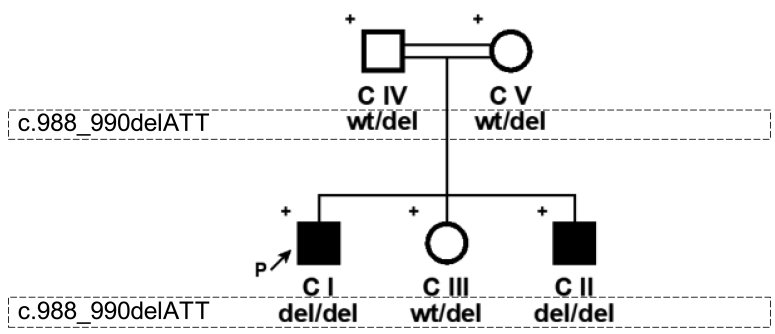

Family B

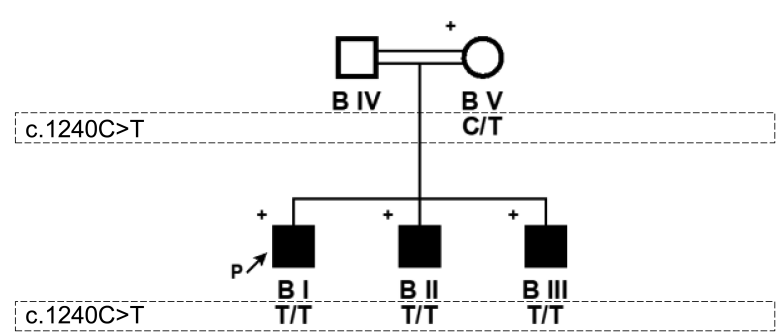

Figure 1. Pedigrees of the families. Plus sign indicates individuals included in the genetic analysis. Arrows show index patients. Variants are defined according to NM_001128303.1. Wt: wild type; del: deletion.

Personal non-commercial use only. The Journal of Rheumatology Copyright @ 2018. All rights reserved. 
Rheumatology classification of juvenile idiopathic arthritis: second revision, Edmonton, 2001. J Rheumatol 2004;31:390-2.

2. Barut K, Adrovic A, Sahin S, Kasapcopur O. Juvenile idiopathic arthritis. Balkan Med J 2017;34:90-101.

3. Manners PJ, Bower C. Worldwide prevalence of juvenile arthritis why does it vary so much? J Rheumatol 2002;29:1520-30.

4. Saurenmann RK, Rose JB, Tyrrell P, Feldman BM, Laxer RM, Schneider R, et al. Epidemiology of juvenile idiopathic arthritis in a multiethnic cohort: ethnicity as a risk factor. Arthritis Rheum 2007;56:1974-84.

5. Glass DN, Giannini EH. Juvenile rheumatoid arthritis as a complex genetic trait. Arthritis Rheum 1999;42:2261-8.

6. Wakil SM, Monies DM, Abouelhoda M, Al-Tassan N, Al-Dusery H, Naim EA, et al. Association of a mutation in LACC1 with a monogenic form of systemic juvenile idiopathic arthritis. Arthritis Rheumatol 2015;67:288-95.

7. Arostegui JI, Rabionet R, Remesal A, Mensa-Vilaro A, Murias S, Alcobendas R, et al. A family carrying a homozygous LACC1 truncated mutation expands the clinical phenotype of this disease beyond systemic-onset juvenile idiopathic arthritis. Pediatr Rheumatol Online J 2015;13 Suppl 1:O76.

8. Kallinich T, Thorwarth A, von Stuckrad SL, Rösen-Wolff A, Luksch $\mathrm{H}$, Hundsdoerfer $\mathrm{P}$, et al. Juvenile arthritis caused by a novel FAMIN (LACC1) mutation in two children with systemic and extended oligoarticular course. Pediatr Rheumatol Online J 2016;14:63.

9. Wallace CA, Ruperto N, Giannini E; Childhood Arthritis and Rheumatology Research Alliance; Pediatric Rheumatology International Trials Organization; Pediatric Rheumatology Collaborative Study Group. Preliminary criteria for clinical remission for select categories of juvenile idiopathic arthritis. J Rheumatol 2004;31:2290-4.

J Rheumatol 2018;45:5; doi:10.3899/jrheum.170834 